\title{
Modulators of the Balance between M1 and M2 Macrophages during Pregnancy
}

\author{
Yong-Hong Zhang ${ }^{1}, \mathrm{Ming} \mathrm{He}^{2}$, Yan Wang ${ }^{2}$ and Ai-Hua Liao ${ }^{1 *}$ \\ ${ }^{1}$ Family Planning Research Institute, Center for Reproductive Medicine, Tongji Medical College, Huazhong University of \\ Science and Technology, Wuhan, P.R. China, ${ }^{2}$ Department of Obstetrics and Gynecology, Maternal and Child Health \\ Hospital of Hubei Province, Wuhan, P.R. China
}

Macrophages are a subset of mononuclear phagocytes of the innate immune system with high plasticity and heterogeneity. At the maternal-fetal interface, macrophages are present in all stages of pregnancy and involved in a variety of activities, including regulation of immune cell activities, decidualization, placental cell invasion, angiogenesis, parturition, and postpartum uterine involution. The activation state and function of uterine-placental macrophages are largely dependent on the local tissue microenvironment. However, disruption of the uterine microenvironment can have profound effects on macrophage activity and subsequently impact pregnancy outcome. Thus, appropriately

\section{OPEN ACCESS}

Edited by:

Charles Dudley Mills, BioMedical Consultants, USA

Reviewed by:

Anthony George Tsolaki, Brunel University London, UK Christian Drouet, Joseph Fourier University, France

*Correspondence: Ai-Hua Liao aihua_liao@sina.com

Specialty section:

This article was submitted to Molecular Innate Immunity, a section of the journal Frontiers in Immunology

Received: 21 October 2016 Accepted: 25 January 2017 Published: 09 February 2017

Citation:

Zhang $Y-H$, He $M$, Wang $Y$ and Liao A-H (2017) Modulators of the

Balance between M1 and M2 Macrophages during Pregnancy.

Front. Immunol. 8:120. doi: 10.3389/fimmu.2017.00120 and timely regulated macrophage polarization has been considered a key determinant of successful pregnancy. Targeting macrophage polarization might be an efficient strategy for maintaining maternal-fetal immune homeostasis and a normal pregnancy. Here, we will review the latest findings regarding the modulators regulating macrophage polarization in healthy pregnancies and pregnancy complications, which might provide a basis for macrophage-centered therapeutic strategies.

Keywords: macrophage, polarization, pregnancy, maternal-fetal interface, GM-CSF, HLA-G, notch signal, Tim-3

\section{INTRODUCTION}

During pregnancy, the maternal immune system is greatly challenged by the semiallogeneic fetus. Instead of immune-mediated rejection, maternal immune adaptation occurs systematically and locally, especially at the maternal-fetal interface. The maternal-fetal interface is a unique microenvironment including three distinct components: the fetal-derived trophoblast, maternal-derived

Abbreviations: APC, antigen-presenting cell; DLL, delta-like; E1, estrone; E, estradiol; E3, estriol; ER, estrogen receptor; EVT, extravillous trophoblast; FR, folate receptor; FTDC, first trimester decidual cell; Gal-9, galectin-9; GM-CSF, granulocyte macrophage colony-stimulating factor; GSI, gamma-secretase inhibitor; hAMSC, human amniotic MSC; HCG, human chorionic gonadotropin; HLA-G, human leukocyte antigen G; iNOS, inducible nitric oxide synthase; IFN- $\gamma$, interferon-gamma; IL, interleukin; IKK, inhibitor of NF-KB kinase; IUGR, intrauterine growth restriction; JAK, janus kinases; JNK, c-Jun N-terminal kinase; LPS, lipopolysaccharides; MAPK, mitogen-activated protein kinase; MHC, major histocompatibility complex; M-CSF,

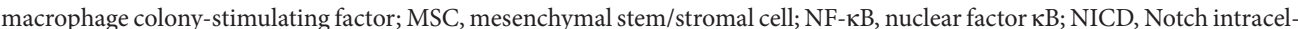
lular domain; NO, nitric oxide; poly(I:C), polyinosinic:cytidylic acid; PE, preelampsia; PGN, peptidoglycan; PTL, preterm labor; ROS, reactive oxygen species; STAT, signal transducers and activators of transcription; TAM, tumor-associated macrophage; $T$. gondii, Toxoplasma gondii; Th, T helper; Tim-3, T-cell immunoglobulin and mucin domain protein 3; TLR, toll-like receptor; TNF- $\alpha$, tumor necrosis factor alpha; TGF- $\beta$, transforming growth factor beta. 
decidual stromal cells, and immune cells. Although the immune cell composition undergoes dramatic changes as gestation progresses, these changes are necessary for maternal-fetal tolerance and healthy pregnancy. Macrophages, approximately $20-25 \%$ of the total decidual leukocytes and the predominant subset of human antigen-presenting cells at the maternal-fetal interface, are in close proximity to the extravillous trophoblast and in the vicinity of spiral arteries. Therefore, they are proposed to be involved in several processes required for a successful pregnancy, including immune tolerance, trophoblast invasion, tissue and vascular remodeling, embryo growth, and initiation of parturition (1). All of these functions are manifestations of macrophage plasticity and heterogeneity, namely, the M1 and M2 subtypes (2). The M1 subtype refers to the classically activated macrophage and displays the capacity to present antigens to the adaptive immune system. With high expression of major histocompatibility complex class II, CD80, CD86, and IL-12, M1-polarized macrophages are more effective at antigen clearance and switching T-cell responses toward T helper-1 immune response (3). Compared to M1 phenotype, M2 populations are alternatively activated. Characterized by typical M2-associated markers (e.g., CD163, CD206, CD209, and IL-10), M2 cells have immunosuppressive capacities, contribute to tissue remodeling, and promote Th2 or antibody-mediated immune responses (4) (Table 1).

Tissue macrophages are deposited during embryonic development of originating from yolk sac cells as early as embryonic day 8.5 and from fetal liver after gastrulation (5). In homeostatic conditions, macrophages are maintained by self-renewal (6). Under inflammatory condition, the embryonically derived macrophages could be partially replaced by bone marrow-derived

TABLE 1 | Phenotype and function of macrophage subsets.



monocytes (7). Macrophages are abundant in the uterus, being the second most abundant endometrial leukocyte population and the predominant myometrial leukocyte population. The numbers of macrophages fluctuate during the estrus cycle and menstrual cycle, which are driven by estrogen and progesterone (8-10). Immediately after copulation, more macrophages are attracted to the endometrium by seminal fluid (11), indicating that a large number of macrophages are necessary to sustain the pregnancy. More evidence for the importance of macrophages was recently provided by Care et al. (12), who reported that specific depletion of macrophages resulted in implantation failure. Furthermore, decidual macrophages are a heterogeneous population with diverse phenotypes that facilitate adaptive responses to the ever-changing environment. Although it has been shown that decidual macrophages do not belong to either of the M1 and M2 subsets (13), some studies have suggested that M2 macrophages or M2 subgroups are the predominant phenotype in the decidua (14).

Pregnancy has been proposed as a dynamic and highly regulated immunologic process (15). Therefore, successful pregnancy requires that the macrophage activation status remains appropriately regulated throughout pregnancy (Figure 1). During the window of the implantation period, macrophages are induced toward M1 activation (16). However, as trophoblasts attach to the endometrial lining and invade the uterine stroma, macrophages switch to a mixed M1/M2 profile (16). The mixed polarization pattern runs through the first trimester and the early phase of the second trimester of pregnancy when the uterine vasculature undergoes remodeling in order to establish an adequate placental-fetal blood supply. After placentation is complete, the macrophages shift toward M2 polarization, which prevents rejection of the fetus and allows fetal growth until parturition. Parturition, which is considered a pro-inflammatory event, is preceded by an accumulation of M1 macrophages in the uterus (17). This inflammatory process promotes the contraction of the uterus, expulsion of the baby, ejection of the placenta, and uterine involution. However, inappropriate macrophage polarization, regardless of when it occurs, is usually associated with abnormal pregnancies, such as spontaneous abortion (18), preterm labor (PTL) (19), preeclampsia (PE) (20), fetal intrauterine growth restriction (IUGR) (21), and intrauterine parasitic infections (22). Therefore, further insight into macrophages would be of great benefit to reproductive immunology (23). However, despite the important roles of macrophages during pregnancy, little is known about the factors responsible for triggering macrophage differentiation and polarization (24). In the current review, we discuss studies that have modulated macrophage polarization in order to provide an overview of potential targets that may promote macrophage homeostasis and normal pregnancy.

\section{MACROPHAGE POLARIZATION}

Macrophage polarization is triggered by signals present in the surrounding environment, accompanied by a set of signaling pathways, transcriptional and posttranscriptional regulatory networks (25). At the most fundamental level, M1/M2 polarity arises 




FIGURE 1 | Dynamics between M1 and M2 macrophages along pregnancy. During the different phases of gestation, macrophages undergo dynamic changes, predominantly displaying the M1 or M2 phenotype. After coitus, granulocyte macrophage colony-stimulating factor levels are increased by transforming growth factor beta (TGF- $\beta$ ) in the seminal fluid and promote M1 activation. In the peri-implantation period, activated M1 macrophages produce inflammatory cytokines and mediators, such as interleukin (IL)-6, IL-1 $\beta$, tumor necrosis factor alpha, and nitric oxide, inducing pro-inflammatory responses and promoting embryo attachment to the decidua. As the trophoblast invades the uterine stroma, decidual macrophages initiate an M1/M2 profile until the early phase of the second trimester of pregnancy, displaying both the pro- and anti-inflammatory phenotype, which endows the host with the ability to promote trophoblast invasion and vascular remodeling and prevent rejection of the embryo. Subsequently, in order to allow fetal development, more progesterone is produced, and an M2-dominant environment is established in the uterus until the end of pregnancy, which includes downregulation of inflammatory mediators, increased generation of antiinflammatory cytokines (e.g., IL-10 and TGF- $\beta$ ), and phagocytosis of apoptotic debris. Finally, M1 macrophages predominate over the M2 subset again during the period of parturition, which is considered an inflammatory event. Accumulated M1 macrophages promote the contraction of the uterus, expulsion of the baby, ejection of the placenta and uterine involution.

from arginine metabolism via two enzymatic pathways [inducible nitric oxide synthase (iNOS) and arginase] that are distinct and antagonistic $(2,26)$. The M1 subtype is a product of the iNOS pathway, whereas M2 is the product of the arginase pathway (2). The factors that determine which pathway is dominant are based on the surrounding signals that the macrophages are exposed to and the available arginine pool $(2,27)$. Therefore, the final activation status of macrophage polarization is ultimately decided by the surrounding milieu. Various surrounding signals participate in macrophage polarization, including adaptive immunity and microorganism-derived molecular patterns, such as lipopolysaccharides (LPS), cytokines, and growth factors released by the injured tissue. Generally, polarized M1 and M2 macrophages are induced and represent the two extremes of a broad spectrum of differentiation states. However, this does not alter their terminal differentiation status. Once M2 macrophages are exposed to M1 signals, or vice versa, "re-polarization" of already differentiated macrophages can occur, which might be more evidence of their highly functional plasticity. Furthermore, this re-education of macrophages is currently under investigation for therapeutic purposes (28). Therefore, a mixed phenotype representing a superposition of the M1 and M2 phenotypes might exist; this was confirmed by findings which suggested that macrophages adopt a mixed phenotype dependent on the relative strength of the stimuli and that cells progress toward an M2 phenotype over time (29). Therefore, macrophage reprogramming by combined activation signals might be dependent on the initial polarization state and doses of stimulation.

Interferon-gamma (IFN- $\gamma$ ), either alone or in combination with other stimulants, including LPS, tumor necrosis factor alpha (TNF- $\alpha$ ), and granulocyte macrophage colony-stimulating factor (GM-CSF), induces M1 macrophage polarization $(4,30)$. In addition, IFN- $\gamma$ and LPS are widely used to induce M1 polarization in vitro. IFN- $\gamma$ induces downstream phosphorylation of signal transducers and activators of transcription-1 (STAT1) by Janus kinases (JAK). LPS specifically activates toll-like receptor (TLR)-4, which can affect the mitogen-activated protein kinase pathway, the interferon regulatory factor pathway, and the nuclear factor $\kappa \mathrm{B}(\mathrm{NF}-\kappa \mathrm{B})$ pathway by inactivating the inhibitor of NF- $\kappa B$ kinase (IKK)-2. Furthermore, the NF- $\kappa B$ pathway has also been implicated in the regulation of STAT1 activity in M1 macrophages. When NF- $\kappa \mathrm{B}$ activity is diminished through deletion of IKK-2, STAT1 activity is enhanced in mouse macrophages (31). The enhanced STAT1 activity subsequently contributes to M1 polarization, with the production of nitric oxide (NO) and the secretion of pro-inflammatory cytokines, such as interleukin (IL)-1 $\beta$, IL-6, IL-12, IL-23, and TNF- $\alpha$ (4, 32). The Notch signaling pathway is also involved in LPS-TLR-4induced expression of inflammatory M1 macrophage cytokines. LPS treatment activates the Notch pathway by a c-Jun N-terminal kinase (JNK)-dependent pathway (33), which enhances NF- $\kappa B$ phosphorylation (34) and pro-inflammatory cytokine secretion $($ IFN- $\gamma$ and TNF- $\alpha$ ) (35).

In contrast, M2 macrophage polarization can be achieved in vitro by macrophage colony-stimulating factor (M-CSF), IL-4, IL-10, IL-13, IL-33, and/or transforming growth factor beta (TGF- $\beta$ ). Both IL-4 and IL-13 activate the JAK-STAT pathway, leading to the activation of STAT6, which is essential for the expression of M2 macrophage markers (36). In M2 macrophages, the production of $\mathrm{NO}$ and pro-inflammatory 
cytokines is diminished, but anti-inflammatory cytokines, such as TGF- $\beta$ and IL-10, are produced. Although the effects of ILs on macrophages are fairly well studied, TGF- $\beta$ remains to be the most puzzling cytokine in regard to its effects on macrophages. Nevertheless, TGF- $\beta$ plays an important role in the pathogenesis of many diseases where macrophages play a key role as well. The relationship between macrophages and TGF- $\beta$ has attracted the attention of researchers since the description of this growth factor (37). Initially, TGF- $\beta$ was found to be a potent immunosuppressive and "macrophage-deactivating" agent (38). Later, the role of TGF- $\beta$ in the function of macrophages was described. The best studied is the role of TGF- $\beta$ in the recruitment and development of tumor-associated macrophages (TAMs) (M2 phenotype) (39, 40 ). It induces an M2-like phenotype by activating the canonical Smad2/3-mediated signaling as well as Smad1/5-mediated signaling (37). Activities of TAM regulated by TGF- $\beta$ stimulate proliferation of tumor cells and lead to tumor immune escape. Therefore, it is clear that understanding of molecular mechanisms of TGF- $\beta$-TAM interaction is highly important for therapeutic targeting of TGF- $\beta$ or macrophages (37).

\section{WHAT MODULATES THE BALANCE BETWEEN M1 AND M2 MACROPHAGES DURING PREGNANCY}

As mentioned above, the unique macrophage phenotype and heterogeneity are important for the establishment and maintenance of a successful pregnancy. The environment in which macrophages mature and differentiate during pregnancy is important for macrophage polarization. Various secreted cytokines, chemokines, growth factors, and hormones, as well as interactions with related cells, are important for macrophages to acquire their unique phenotype and function (41) (Figure 2).

\section{GM-CSF AND M-CSF}

Granulocyte macrophage colony-stimulating factor and M-CSF belong to the CSF family and are major differentiation growth factors that mediate M1 and M2 polarization, respectively. Both GM-CSF and M-CSF as well as their receptors are present at the maternal-fetal interface. The endometrium, decidua, and trophoblast are the main sources of CSFs. Their receptors have been observed in both murine and human female trophoblasts and reproductive tracts (42-46). The membrane receptors have also been identified on endometrial immune cells, such as macrophages, granulocytes, and dendritic cells (42). Endometrial GM-CSF production was shown to be positively regulated by estrogen and inhibited by progesterone (47). With coitus, GM-CSF expression is increased by TGF- $\beta$ in the male seminal fluid (48). This surge in GM-CSF production together with other cytokines and chemokines induced by seminal fluid triggers a controlled inflammatory response within the decidua associated with an influx of macrophages, which maintains a mild proinflammatory phenotype around the time of embryo implantation (49). Svensson et al. $(50,51)$ found that trophoblast-derived M-CSF polarized maternal monocytes toward M2 macrophages with a resemblance to decidual macrophages during the first trimester of normal pregnancy. GM-CSF-stimulated macrophages presented a phenotype that was more similar to that of macrophages activated by LPS and IFN $-\gamma$, namely, M1 activation (50). Among the Th2 cytokines (e.g., IL-4, IL-13), only IL-10 was able to overcome the effect of GM-CSF during macrophage polarization. Therefore, trophoblast-derived M-CSF and IL-10 induce the polarization of decidual macrophages, contributing to the homeostatic and tolerant immune environment required for successful fetal development. Apart from the trophoblast, first trimester decidual cells (FTDCs) are another source of M-CSF and GM-CSF. Li et al. (52) found that FTDC-secreted $\mathrm{M}-\mathrm{CSF}$ induced decidual immune tolerance by switching to M2 macrophage polarization and phagocytic capacity in response to pro-inflammatory stimuli. However, excessive pro-inflammatory cytokines, such as IL- $1 \beta$ and TNF- $\alpha$, markedly enhance GM-CSF expression in FTDC, which subsequently polarizes macrophages toward the M1 subtype in PE (53-55). Conversely, enhanced expression of GM-CSF might contribute to PE by promoting M1 polarization.

\section{PREGNANCY-RELATED HORMONES}

Apart from immune adaptation, pregnancy is marked by significant temporal changes of a variety of hormones throughout gestation. Therefore, the success of pregnancy might depend on a synchronized immune-endocrine crosstalk at the maternal-fetal interface (56). Hormones are important in terms of maintenance of the suitable environment and sufficient nutrition for the developing fetus. Hormones modulate both innate and adaptive immune cells to adopt to fetal development. Therefore, maternal tolerance to the semiallogeneic fetus is achieved in concert with a variety of endocrine stimulations. Estrogens, progesterone, and human chorionic gonadotropin (HCG) are three of the main hormones during pregnancy. These hormones have recently been proposed to modulate macrophage polarization during pregnancy.

Estrogens are a group of compounds known for their importance in the estrus cycle of humans and other animals. Three main common estrogens are present throughout pregnancy, including estrone (E1), estradiol (E2), and estriol (E3). The placenta is the primary site of E1 and E2 production, and it converts 16-hydroxydehydroepiandrosterone to E3. With estrogen receptors (ERs) expressed in lymphocytes, macrophages, and dendritic cells, estrogens contribute to fetal tolerance by regulating the phenotype and function of different immune cell populations (57). E2 occurs in high concentrations in non-pregnant as well as pregnant females and is responsible for the majority of the "classic" estrogenic effects in reproductive tissues. E2 has bipotential effects on macrophages, with low concentrations promoting proinflammatory cytokine production (e.g., IL-1 $\beta$, IL-6, and TNF- $\alpha$ ) and high doses reducing secretion of these cytokines (58). This finding may indicate that E2 can regulate macrophage polarization to some extent. E3 is produced in high concentrations by the fetoplacental unit during pregnancy and accounts for almost $90 \%$ of all estrogens produced during pregnancy (59). However, the immunological effects of E3 have not been well characterized, 


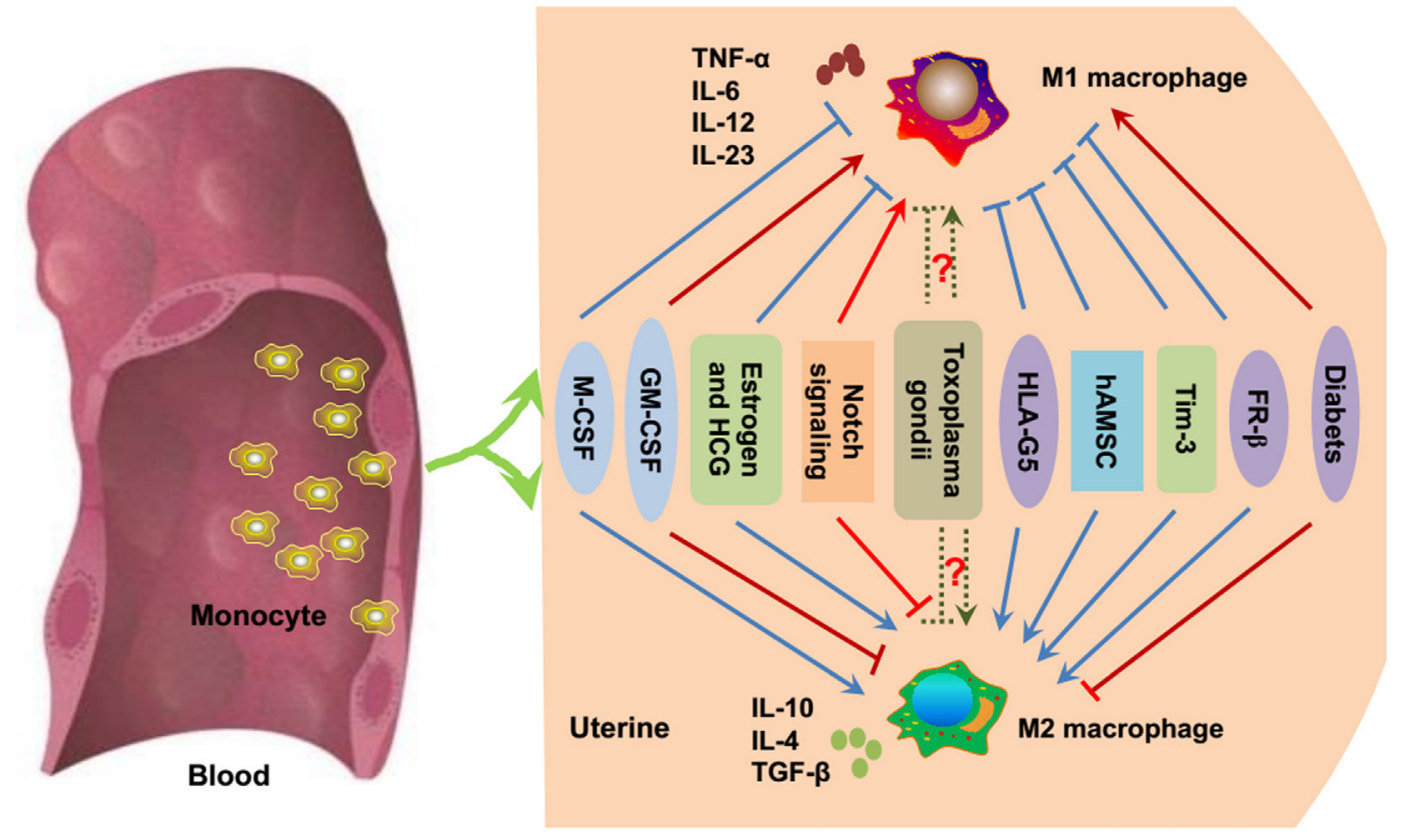

FIGURE 2 | Essential modulators of macrophage polarization during pregnancy. Under inflammatory conditions, bone marrow-derived monocytes contribute to tissue macrophage homeostasis. As previously mentioned, M-CSF, estrogen, HCG, HLA-G5, hAMSC, Tim-3, and FR- $\beta$ promote the polarization toward M2 macrophages (blue point arrows) and inhibit M1 polarization (blue block arrows). GM-CSF, Notch signaling, and diabetes/hyperglycemia have been implicated in the polarization of M1 macrophages (red point arrows), while suppressing M2 macrophage polarization (red block arrows). Whether Toxoplasma gondii facilitates M1 or M2 macrophage polarization is uncertain (dashed green arrows), and it mainly depends on the host immune status and the virulence of the pathogen. M-CSF, macrophage colony-stimulating factor; GM-CSF, granulocyte macrophage colony-stimulating factor; HCG, human chorionic gonadotropin; HLA-G, human leukocyte antigen G; hAMSC, human amniotic mesenchymal stem cell; Tim-3, T-cell Ig and mucin domain protein 3; FR- $\beta$, folate receptor $\beta$.

and it is assumed that the effects of E3 are broadly the same as E2 because both estrogens signal through the same ERs (60).

Progesterone is produced by the corpus lutea in the ovaries in non-pregnant females. After conception, progesterone is produced by the corpus luteum until 5-6 weeks of gestation, and then after the 12th week of gestation, the placenta becomes the dominant producer of progesterone. Progesterone has various functions, such as promoting endometrial decidualization for embryo implantation (61), inhibiting smooth muscle contractility, and maintaining myometrial quiescence (62). Additionally, progesterone is considered to be anti-inflammatory. Progesterone receptors have been identified in macrophages, and progesterone can inhibit nitrite and NO production as well as TNF- $\alpha$ expression by murine macrophages (63). However, macrophage polarization might not be influenced by progesterone during pregnancy. Furcron et al. (64) found that vaginal progesterone treatment had anti-inflammatory effects at the murine maternal-fetal interface. Inflammation has been implicated in physiological (65) and pathological parturition (66), such as PTL. Therefore, the effects of progesterone in the prevention of PTL may be mediated by its anti-inflammatory capacity (64). Although vaginal progesterone reduces the proportion of decidual macrophages, it does not result in M1 $\rightarrow$ M2 macrophage polarization in murine models. However, whether progesterone modulates macrophage polarization in human pregnancy is still unclear.
Human chorionic gonadotropin is the first hormone that participates in the interactions between the mother and the fetus. HCG is a heterodimeric glycoprotein, which is initially produced in the developing placenta after conception and later by the placental component syncytiotrophoblast (67). Temporal fluctuations in the production of HCG are marked by its maximal levels by the 10th week of pregnancy, and then it falls slowly to the lowest point at 17 weeks and remains at a low but readily measurable level for the remainder of the pregnancy (68). HCG receptors are widely expressed in reproductive tissues (69), maternal-fetal tissues $(70)$, and immune cells $(71,72)$. Therefore, HCG is thought to be involved in preserving the progesteroneproducing corpus luteum (73), promoting angiogenesis (74) and trophoblast differentiation (75) and maintaining myometrial quiescence (76) and maternal-fetal tolerance (77). Macrophages express HCG receptors throughout gestation (78). HCG treatment of IFN- $\boldsymbol{\gamma}$-primed macrophages resulted in increased production of NO, reactive oxygen species, and IL-6 and enhanced phagocytosis of apoptotic cells (71). Therefore, HCG can enhance macrophage function (71). At the maternal-fetal interface during late gestation, HCG has anti-inflammatory effects and prevents endotoxin-induced PTL but causes dystocia and fetal compromise in mice (79). Unlike progesterone, HCG treatment reduces the proportion of macrophages at the maternal-fetal interface but induces M1 $\rightarrow$ M2 macrophage polarization (79). 


\section{NOTCH SIGNALING}

The Notch signaling pathway is evolutionarily conserved and is involved in regulating cell proliferation, apoptosis, and cell fate decisions during development and adult tissue homeostasis (80). In mammals, there are four Notch receptors (Notch 1-4) and five distinct ligands [Jagged1, Jagged2, Delta-like 1 (DLL1), DLL3, and DLL4]. The interaction between Notch ligands and receptors leads to proteolytic cleavage of the receptor and liberates the Notch intracellular domain (NICD) from the membrane. Then, NICD transfers to the nucleus, where it activates the recombining binding protein suppressor, subsequently allowing the recruitment of coactivators and leading to the transcription of Notch genes. Notch signaling has been proven to determine the fate of immune cells and is involved in T- and B-cell activation (81) and macrophage polarization toward M1 cells (82). Notch signaling is also involved in the polarization of decidual macrophages. Notch signaling pathways exert effects throughout pregnancy and are activated in response to TLR ligands (83). PTL can be induced in animal models by pathogen-derived TLR ligands for TLR4 (LPS), TLR2 (peptidoglycan, PGN), and TLR3 [polyinosinic:cytidylic acid, poly(I:C)] as well as in a synergistic manner (TLR2 + TLR3). Altered expression of Notch signaling-related molecules was closely associated with LPS-induced PTL but not in hormonally induced PTL (83). In the decidua of LPS-induced PTL, macrophage polarization is skewed toward M1 cells, and this process is dependent on the activation of Notch signaling. Furthermore, PGN + poly(I:C) administration induces the expression of DLL-1 and Notch 1 in decidual macrophages, which are double positive for CD11c (M1 marker) and CD206 (M2 marker), with the generation of both M1-associated cytokines (IL-6, TNF- $\alpha$ ) and M2-associated cytokines (IL-10). However, lower secretion of both M1- and M2-associated cytokines was observed by the Notch inhibitor gamma-secretase inhibitor (84). Therefore, upregulated Notch-related inflammation may be associated with inflammation-induced PTL by regulating macrophage polarization.

\section{Toxoplasma gondii}

Toxoplasma gondii infection is the leading cause of fetal IUGR among the five pathogens termed TORCH (including Toxoplasma, rubella virus, cytomegalovirus, and herpes virus and other pathogens). T. gondii infection may result in congenital toxoplasmosis, miscarriage, stillbirth, and increased pregnancy complications (85). All these abnormal pregnancy outcomes may result from immune imbalances induced by T. gondii (86). Macrophages are important effector cells for the control and killing of intracellular T. gondii, and they also serve as longterm host cells for the replication and survival of the parasite (87). These different outcomes might depend on macrophage activation (M1 or M2) after T. gondii infection. Jensen et al. (88) found that macrophages infected with the type I and type III $T$. gondii polarized to M1 activation, while type II infection skewed to M2 polarization in pregnant mice. Moreover, the ability of Toxoplasma to induce specific macrophage activation could be associated with consequences on virulence, local parasite burden, and inflammatory-related pathology. For example, macrophages infected with the TgCtwh6 strain (type I with low virulence) were preferentially biased toward M1 activation and an increased trophoblast apoptosis index in vitro (22), while TgCtwh3 (type I with high virulence)-infected macrophages were polarized toward M2 activation. However, higher apoptosis levels of trophoblasts were found in TgCtwh3 infection in vivo, which might have resulted from further Th2 bias by TgCtwh3, subsequently promoting parasite duplication (22).

Parasite-derived factors, ROP16 and GRA15, work independently to achieve M1 and M2 activation (22). ROP16, a rhoptry protein, has serine-threonine kinase activity (89) and induces M2 activation through the STAT6 pathway, while GRA15 drives macrophages to M1 polarization via NF- $\mathrm{KB}$ activation. Further investigations showed that the induction of macrophage polarization depends on polymorphisms of the two proteins in strains with different genotypes $(90,91)$. The phosphokinase ROP16/III allelic variation of leucine but not serine at 503 is responsible for M2 polarization, while GRA15 II promotes M1 polarization (92). Differing from the archetypal lineages of type I, II, and III circulating in Northern America and Europe, type Chinese 1 is the predominant clonal lineage in China. The Wh6 strain of Chinese 1 has comparatively low virulence to mice (93). Sequencing of the effectors of ROP16 and GRA15 showed that Wh6 strain possesses the allelic polymorphisms of the two effector molecules (ROP16 $6_{\text {I/III }} 503 \mathrm{~L}$ and GRA15 1 II), suggesting a different mechanism of macrophage-biased induction in Chinese 1 strain infection (85). Rats with acute Wh6 infection prior to pregnancy-favored M1 polarization, accompanied by an increased proportion of fetal IUGR, inflammatory scores of the placenta, and reduced numbers of embryos (85). These findings strongly suggest the association of M1-biased immunity induced by Toxoplasma infection on gestation with the consequence of immunopathology and adverse pregnancy outcomes. However, an M2 bias was observed in acute infection after gestation, indicating that part or most of the macrophages might be induced to $\mathrm{M} 2$ in the microenvironment during pregnancy, and the Th2-dominant immune response in pregnant rats somewhat inhibits the excessive bias of the macrophages toward M1. Most of these findings were observed in pregnant rats infected with $T$. gondii, and the macrophages were obtained from the peritoneal cavity. The relationship between T. gondii infection and macrophage polarization during human pregnancy is still unclear.

\section{HUMAN LEUKOCYTE ANTIGEN G}

Human leukocyte antigen G belongs to HLA class Ib. There are seven isoforms of HLA-G; G1-4 are membrane bound, whereas G5-7 are soluble proteins (94). Both membrane-bound and soluble HLA-G molecules are detected in human placentas, decidua, and maternal blood (95). During healthy pregnancy, the plasma level of soluble HLA-G5 increases in the first trimester and gradually declines as the pregnancy advances (96). A decreased or undetectable level of soluble HLA-G in the maternal circulation during the first/second trimester is associated with complications, such as recurrent spontaneous abortion, PE, and 
IUGR $(97,98)$. Soluble HLA-G5 participates in immune tolerance under physiological (pregnancy) and pathological (tumor and allograft) conditions. Therefore, decidual macrophage maturation and differentiation might be regulated by HLA-G5, which could be released from the trophoblast, as its functional receptors are expressed in monocytes and decidual macrophages (94, 98). Lee et al. (99) demonstrated that soluble HLA-G5 polarized macrophages toward the M2 phenotype, with higher phagocytic activity and increased IDO expression, suppressing IFN- $\gamma$ expression in T-cells and promoting trophoblast invasion. Therefore, these findings suggest a role for soluble HLA-G5 in driving macrophage polarization into the decidual macrophagelike phenotype, which promotes maternal-fetal tolerance and placental development. The potential molecular mechanisms that regulate the soluble HLA-G5-polarized macrophages in maternal-fetal tolerance and placental remodeling are still unclear.

\section{AMNIOTIC MESENCHYMAL STROMAL CELL}

Mesenchymal stem/stromal cells (MSCs), derived from both maternal and fetal compartments, strongly contribute to maternal-fetal tolerance, mainly resulting from their broad immune regulatory capacities (100-102). The immune regulatory properties of human amniotic MSCs (hAMSCs) are the subject of growing interest $(103,104)$. In addition to T lymphocytes, hAMSCs also act on the monocyte/macrophage lineage regulating their activation (105-107). Interestingly, hAMSCs promote monocyte differentiation into anti-inflammatory M2 cells (107). Indeed, hAMSCs from a normal pregnancy block M1 differentiation and switch them to M2 cells (108). Therefore, it is reasonable to speculate that the immune-modulatory properties of hAMSCs are altered and contribute to the development of abnormal pregnancies, such as PE. However, no intrinsic impairment of hAMSCs was found between healthy pregnancy and PE (108). These results suggest that hAMSCs might not contribute to the development of PE but conversely, could participate in offsetting the inflammatory status that characterizes PE.

\section{T-CELL IMMUNOGLOBULIN AND MUCIN DOMAIN PROTEIN 3 (TIM-3)}

T-cell immunoglobulin and mucin domain protein 3 was first described as a molecule specifically expressed on the surface of IFN- $\gamma$-producing Th1 and cytotoxic T-1 cells (109). The engagement of Tim-3 with its ligand, galectin-9, could induce the exhaustion or apoptosis of effector $\mathrm{T}$ cells, and thus might regulate immune tolerance (110). In addition to being expressed on activated T-cells, Tim-3 is constitutively expressed on cells of the innate immune system in both mice and humans, and Tim-3 expression is enhanced in M2 macrophages $(111,112)$. Recent data have demonstrated that Tim-3 regulates innate immune cells to induce maternal-fetal tolerance $(113,114)$. Chabtini et al. (114) found that blockade of Tim-3 by RMT3-23 (antiTim-3) antibody resulted in accumulation of macrophages at the maternal-fetal interface and upregulation of pro-inflammatory cytokines. Furthermore, Tim-3 blockade during early pregnancy inhibits the phagocytic potential of macrophages, resulting in the accumulation of apoptotic bodies at the maternal-fetal interface. This accumulation elicits local immune responses, leading to the abrogation of tolerance at the maternal-fetal interface and fetal rejection. All these findings suggest that Tim-3 blockade during the first trimester skews macrophages toward M1 activation rather than M2 polarization.

\section{FOLATE RECEPTOR $\beta$}

Hofbauer cells are macrophages that reside within the mesenchymal stroma of the chorionic villi (115), which are thought to be of fetal origin (116). Constitutive expression of CD209 and high levels of CD163, CD45, HLA-A, HLA-B, HLA-C, IL-10, and TGF- $\beta$ suggest that Hofbauer cells skew toward M2 in healthy pregnancies $(117,118)$. Therefore, Hofbauer cells may participate in placental angiogenesis, tissue remodeling, and modulation of inflammation-like decidual macrophages (118). Folate receptors (FRs) are glycoproteins responsible for high affinity folate binding and subsequent transport into cells via endocytosis. The FR family includes three types: FR- $\alpha$, FR- $\beta$, and FR- $\gamma / \gamma^{\prime}$. The expression profile of each FR subtype depends on differentiation stage and tissue type. FR- $\beta$ is expressed on a number of hematopoietic precursor cells and myelomonocytic lineages. However, it usually stays in an inactive form, unable to bind folate. Functional FR- $\beta$ is detected on activated macrophages in the placenta. Moreover, it is preferentially expressed on M2 macrophages and is considered a biomarker for M2 macrophages (119). Decreased expression of FR- $\beta$ and CD163 has been observed in Hofbauer cells from women with PE (120). These findings indicate that Hofbauer cells might switch toward M1 polarity in PE, then M1 macrophages might contribute to the development of PE.

\section{DIABETES/HYPERGLYCEMIA}

Diabetes impairs fetal development and increases the risk of metabolic disorders in adulthood. Strong changes in the expression of placental genes related to markers and mediators of inflammation are elicited by diabetes (121). Compared with healthy pregnant women, Hofbauer cells exhibit an M1-like phenotype and function in women with diabetes (122). Further investigation proved that diabetes and/or hyperglycemia could switch Hofbauer cells from the M2 to M1 phenotype in vivo and in vitro. Therefore, the altered functional phenotype of Hofbauer cells might contribute to the detrimental inflammation status of the placenta and eventually result in negative consequences to fetal development. Although the underlying mechanism is still unclear, there might be an assumption that the shift from the M2 to the M1 profile might involve another higher level of communication between environmental stimuli and cell responses, such as epigenetic modifications.

In addition, other factors modulating macrophage polarization have been found in other immune disorders (123-126), such as programmed cell death 1, IRGM, and miRNAs. However, whether these modulators contribute to macrophage homeostasis during pregnancy has not been characterized. 


\section{THE BRIGHT FUTURE OF MACROPHAGE- ORIGINATED THERAPY FOR PREGNANCY COMPLICATIONS}

It is now known that macrophage polarization governs the fate of an organ (127). As discussed above, accurately regulated uterine macrophage polarization, namely, the M1/M2 balance, is involved in the establishment, maintenance, and termination of normal pregnancy. However, an M1/M2 imbalance results in pregnancy loss or pregnancy complications. Thus, the realization that macrophages play a pivotal role in directing pregnancy outcomes, either directly or by influencing $\mathrm{T}$ - and B-cell functions, is opening novel approaches to pregnancy complications using immunotherapy.

Modulating macrophage polarization, namely, converting the M1-type macrophages into M2-type macrophages, might be a breakthrough that will facilitate successful immunotherapy. Although no studies have focused on pregnancy, the combination of 5-Aza 2-deoxycytidine and Trichostatin A, two epigenetic modifiers, decreased expression of the M1 phenotype while augmenting expression of the M2 phenotype in LPS-induced macrophages (128). It has also been found that a shift toward M2-like macrophages protects against structural and functional damage in adriamycin-induced nephropathy in SCID mice (129).

Although the abovementioned modulators of macrophage polarization have only recently received attention from researchers, they do have a promising future. Using the abovementioned studies as examples, more studies utilizing modulators to manipulate the pathogenesis of disorders should be undertaken. Given that those modulators contribute to the development of inflammatory disorders, they could also serve as more effective therapeutic approaches (127). Furthermore, we believe that macrophage-based immunotherapy will help ameliorate

\section{REFERENCES}

1. Erlebacher A. Immunology of the maternal-fetal interface. Annu Rev Immunol (2013) 31:387-411. doi:10.1146/annurev-immunol-032712100003

2. Mills CD. Macrophage arginine metabolism to ornithine/urea or nitric oxide/citrulline: a life or death issue. Crit Rev Immunol (2001) 21(5):399-425. doi:10.1615/CritRevImmunol.v21.i5.10

3. Mills CD, Kincaid K, Alt JM, Heilman MJ, Hill AM. M-1/M-2 macrophages and the Th1/Th2 paradigm. J Immunol (2000) 164(12):6166-73. doi:10.4049/ jimmunol.164.12.6166

4. Mantovani A, Sica A, Sozzani S, Allavena P, Vecchi A, Locati M. The chemokine system in diverse forms of macrophage activation and polarization. Trends Immunol (2004) 25(12):677-86. doi:10.1016/j.it.2004.09.015

5. Sheng J, Ruedl C, Karjalainen K. Most tissue-resident macrophages except microglia are derived from fetal hematopoietic stem cells. Immunity (2015) 43(2):382-93. doi:10.1016/j.immuni.2015.07.016

6. Yona S, Kim KW, Wolf Y, Mildner A, Varol D, Breker M, et al. Fate mapping reveals origins and dynamics of monocytes and tissue macrophages under homeostasis. Immunity (2013) 38(1):79-91. doi:10.1016/j. immuni.2012.12.001

7. Epelman S, Lavine KJ, Beaudin AE, Sojka DK, Carrero JA, Calderon B, et al. Embryonic and adult-derived resident cardiac macrophages are maintained through distinct mechanism at steady state and during inflammation. Immunity (2014) 40(1):91-104. doi:10.1016/j.immuni.2013.11.019 inflammatory disorders via more natural, effective, and less-toxic and disabling means than drugs or surgery.

\section{CONCLUSION}

Macrophages play important roles in embryo implantation, placentation, pregnancy maintenance, and initiation of parturition. With high plasticity, their phenotypes and functions are influenced by the microenvironment. Accurate regulation of macrophage polarization is required for successful pregnancy. Otherwise, pregnancy complications and poor outcomes occur with ill-timed or ill-placed macrophage polarization. Although several factors regulating M1 versus M2 polarization during pregnancy have been found, such as growth factors, hormones, infection, and Tim-3, numerous questions remain: (i) What are the specific M1 and M2 factors and their roles in human pregnancy? (ii) Since M2 macrophages facilitate pregnancy maintenance, how is this accomplished at the molecular and cellular levels? (iii) How do polarized macrophages influence pregnancy at the cellular and molecular levels? (iv) As pregnancy is characterized by multiple stages, are there any specific factors modulating macrophage polarization at specific stages? and most importantly, (v) Can small molecules be developed to switch or regulate macrophage polarization?

\section{AUTHOR CONTRIBUTIONS}

Y-HZ: design, text and drawings. $\mathrm{MH}$ and YW: text. A-HL: design, text revision and final approval.

\section{FUNDING}

This work was supported by research grants from the National Natural Science Foundation of China (No. 81671490).

8. De M, Wood GW. Influence of oestrogen and progesterone on macrophage distribution in the mouse uterus. JEndocrinol (1990) 126:417-24. doi:10.1677/joe.0.1260417

9. Bulmer JN, Morrison L, Longfellow M, Ritson A, Pace D. Granulated lymphocytes in human endometrium: histochemical and immunohistochemical studies. Hum Reprod (1991) 6:791-8.

10. Klentzeris LD, Bulmer JN, Warren A, Morrison L, Li TC, Cooke ID. Endometriallymphoid tissue in the timed endometrial biopsy: morphometric and immunohistochemical aspects. Am J Obstet Gynecol (1992) 167:667-74. doi:10.1016/S0002-9378(11)91568-3

11. Roertson SA, Mau VJ, Tremellen KP, Seamark RF. Role of high molecular weight seminal vesicle proteins in eliciting the uterine inflammatory response to semen in mice. J Reprod Fertil (1996) 107:265-77. doi:10.1530/ jrf.0.1070265

12. Care AS, Diener KR, Jasper MJ, Brown HM, Ingman WV, Robertson SA. Macrophages regulate corpus luteum development during embryo implantation in mice. JClin Invest (2013) 123:3472-87. doi:10.1172/ JCI60561

13. Houser BL, Tilburgs T, Hill J, Nicotra ML, Strominger JL. Two unique human decidual macrophage populations. J Immunol (2011) 186:2633-42. doi:10.4049/jimmunol.1003153

14. Gustafsson C, Mjösberg J, Matussek A, Geffers R, Matthiesen L, Berg G, et al. Gene expression profiling of human decidual macrophages: evidence for immunosuppressive phenotype. PLoS One (2008) 3:e2078. doi:10.1371/ journal.pone.0002078 
15. Mor G, Cardenas I, Abrahams V, Guller S. Inflammation and pregnancy: the role of the immune system at the implantation site. Ann N Y Acad Sci (2011) 1221(March):80-7. doi:10.1111/j.1749-6632.2010.05938.x

16. Jaiswal MK, Mallers TM, Larsen B, Kwak-Kim J, Chaouat G, Gilman-Sachs A, et al. V-ATPase upregulation during early pregnancy: a possible link to establishment of an inflammatory response during preimplantation period of pregnancy. Reproduction (2012) 143(5):713-25. doi:10.1530/REP$12-0036$

17. Hamilton S, Oomomian Y, Stephen G, Shynlova O, Tower CL, Garrod A, et al. Macrophages infiltrate the human and rat decidua during term and preterm labor: evidence that decidual inflammation precedes labor. Biol Reprod (2012) 86(2):39. doi:10.1095/biolreprod.111.095505

18. Guenther S, Vrekoussis T, Heublein S, Bayer B, Anz D, Knabl J, et al. Decidual macrophages are significantly increased in spontaneous miscarriages and over-express FasL: a potential role for macrophages in trophoblast apoptosis. Int J Mol Sci (2012) 13(7):9069-80. doi:10.3390/ijms13079069

19. Diamond AK, Sweet LM, Oppenheimer KH, Bradley DF, Phillippe M. Modulation of monocyte chemotactic protein-1 expression during lipopolysaccharide-induced preterm delivery in the pregnant mouse. Reprod Sci (2007) 14(6):548-59. doi:10.1177/1933719107307792

20. Schonkeren D, van der Hoorn ML, Khedoe P, Swings G, van Beelen E, Claas F, et al. Differential distribution and phenotype of decidual macrophages in preeclamptic versus control pregnancies. Am J Pathol (2011) 178(2):709-17. doi:10.1016/j.ajpath.2010.10.011

21. Prins JR, Faas MM, Melgert BN, Huitema S, Timmer A, Hylkema MN, et al. Altered expression of immune-associated genes in first-trimester human decidua of pregnancies later complicated with hypertension or foetal growth restriction. Placenta (2012) 33(5):453-5. doi:10.1016/j.placenta. 2012.02.010

22. Liu T, Zhang Q, Liu L, Xu X, Chen H, Wang H, et al. Trophoblast apoptosis through polarization of macrophages induced by Chinese Toxoplasma gondii isolates with different virulence in pregnant mice. Parasitol Res (2013) 112(8):3019-27. doi:10.1007/s00436-013-3475-3

23. PrabhuDas M, Bonney E, Caron K, Dey S, Erlebacher A, Fazleabas A, et al. Immune mechanisms at the maternal-fetal interface: perspectives and challenges. Nat Immunol (2015) 16(4):328-34. doi:10.1038/ni.3131

24. Brown MB, von Chamier M, Allam AB, Reyes L. M1/M2 macrophage polarity in normal and complicated pregnancy. Front Immunol (2014) 5:606. doi:10.3389/fimmu.2014.00606

25. Wang $\mathrm{N}$, Liang $\mathrm{H}$, Zen $\mathrm{K}$. Molecular mechanisms that influence the macrophage m1-m2 polarization balance. Front Immunol (2014) 5:614. doi:10.3389/fimmu.2014.00614

26. Mills CD. M1 and M2 macrophages: oracles of health and disease. Crit Rev Immunol (2012) 32(6):463-88. doi:10.1615/CritRevImmunol.v32.i6.10

27. Comalada M, Yeramian A, Modolell M, Lloberas J, Celada A. Arginine and macrophage activation. Methods Mol Biol (2012) 844:223-35. doi:10.1007/ 978-1-61779-527-5_16

28. Mantovani A, Allavena P. The interaction of anticancer therapies with tumor-associated macrophages. J Exp Med (2015) 212(4):435-45. doi:10.1084/jem.20150295

29. Smith TD, Tse MJ, Read EL, Liu WF. Regulation of macrophage polarization and plasticity by complex activation signals. Integr Biol (Camb) (2016) 8(9):946-55. doi:10.1039/c6ib00105j

30. Lawrence T, Natoli G. Transcriptional regulation of macrophage polarization enabling diversity with identity. Nat Rev Immunol (2011) 11(11):750-61. doi:10.1038/nri3088

31. Fong CH, Bebien M, Didierlaurent A, Nebauer R, Hussell T, Broide D, et al. An antiinflammatory role for IKKbeta through the inhibition of "classical" macrophage activation. J Exp Med (2008) 205(6):1269-76. doi:10.1084/ jem.20080124

32. Soler C, Felipe A, Garcia-Manteiga J, Serra M, Guillen-Gomez E, Casado FJ, et al. Interferon-gamma regulates nucleoside transport systems in macrophages through signal transduction and activator of transduction factor 1 (STAT1)-dependent and -independent signalling pathways. Biochem J (2003) 375(Pt3):777-83. doi:10.1042/bj20030260

33. Tsao PN, Wei SC, Huang MT, Lee MC, Chou HC, Chen CY, et al. Lipopolysaccharide-induced Notch signaling activation through JNKdependent pathway regulates inflammatory response. J Biomed Sci (2011) 18:56. doi:10.1186/1423-0127-18-56
34. Cao Q, Li P, Lu J, Dheen ST, Kaur C, Ling EA. Nuclear factor-kappaB/p65 responds to changes in the Notch signaling pathway in murine BV-2 cells and in amoeboid microglia in postnatal rats treated with the gamma-secretase complex blocker DAPT. J Neurosci Res (2010) 88(12):2701-14. doi:10.1002/ jnr.22429

35. Monsalve E, Perez MA, Rubio A, Ruiz-Hidalgo MJ, Baladron V, GarciaRamirez JJ, et al. Notch-1 up-regulation and signaling following macrophage activation modulates gene expression patterns known to affect antigen-presenting capacity and cytotoxic activity. J Immunol (2006) 176(9):5362-73. doi:10.4049/jimmunol.176.9.5362

36. Ishii M, Wen H, Corsa CA, Liu T, Coelho AL, Allen RM, et al. Epigenetic regulation of the alternatively activated macrophage phenotype. Blood (2009) 114(15):3244-54. doi:10.1182/blood-2009-04-217620

37. Gratchev A. TGF- $\beta$ signalling in tumor associated macrophages. Immunobiology (2017) 222(1):75-81. doi:10.1016/j.imbio.2015.11.016

38. Tsunawaki S, Sporn M, Ding A, Nathan C. Deactivation of macrophages by transforming growth factor-beta. Nature (1988) 334(6179):2602. doi:10.1038/334260a0

39. Wahl SM, Hunt DA, Wakefield LM, McCartney-Francis N, Wahl LM, Roberts AB, et al. Transforming growth factor type beta induces monocyte chemotaxis and growth factor production. Proc Natl Acad Sci U S A (1987) 84(16):5788-92. doi:10.1073/pnas.84.16.5788

40. Bombara C, Ignotz RA. TGF-beta inhibits proliferation of and promotes differentiation of human promonocytic leukemia cells. J Cell Physiol (1992) 153(1):30-7. doi:10.1002/jcp.1041530106

41. Ning F, Liu H, Lash GE. The role of decidual macrophages during normal and pathological pregnancy. Am J Reprod Immunol (2016) 75(3):298-309. doi:10.1111/aji.12477

42. Arceci RJ, Shanahan F, Stanley ER, Pollard JW. Temporal expression and location of colony-stimulating factor 1 (CSF-1) and its receptor in the female reproductive tract are consistent with CSF-1-regulated placental development. Proc Natl Acad Sci U S A (1989) 86:8818-22. doi:10.1073/ pnas.86.22.8818

43. Croy BA, Guilbert LJ, Brown MA, Gough NM, Stinchcomb DT, Reed $\mathrm{N}$, et al. Characterization of cytokine production by the metrial gland and granulated metrial gland cells. J Reprod Immunol (1991) 19:149-66. doi:10.1016/0165-0378(91)90014-H

44. Robertson SA, O'Connell AC, Hudson SN, Seamark RF. Granulocyte macrophage colony-stimulating factor (GM-CSF) targets myeloid leukocytes in the uterus during the post-mating inflammatory response in mice. J Reprod Immunol (2000) 46:131-54. doi:10.1016/S0165-0378(99)00060-1

45. Kanzaki H, Crainie M, Lin H, Yui J, Guilbert LJ, Mori T, et al. The in situ expression of granulocyte-macrophage colony-stimulating factor (GM-CSF) mRNA at the maternal-fetal interface. Growth Factors (1991) 5:69-74. doi:10.3109/08977199109000272

46. Giacomini G, Tabibzadeh SS, Satyaswaroop PG, Bonsi L, Vitale L, Bagnara GP, et al. Epithelial cells are the major source of biologically active granulocyte macrophage colony-stimulating factor in human endometrium. Hum Reprod (1995) 10:3259-63.

47. Robertson SA, Mayrhofer G, Seamrk RF. Ovarian steroid hormones regulate granulocyte-macrophage colony-stimulating factor synthesis by uterine epithelial cells in the mouse. Biol Reprod (1996) 54:183-96. doi:10.1095/ bioreprod54.1.183

48. Tremellen KP, Seamark RF, Robertson SA. Seminal transformation growth factor beta 1 stimulates granulocyte-macrophage colony-stimulating factor production and inflammatory cell recruitment in the murine uterus. Biol Reprod (1998) 58:1217-35. doi:10.1095/biolreprod58.5.1217

49. McMaster MT, Newton RC, Dey SK, Andrews GK. Activation and distribution of inflammatory cells in the mouse uterus during the preimplantation period. J Immunol (1992) 15:1699-705.

50. Svensson J, Jenmalm MC, Matussek A, Geffers R, Berg G, Ernerudh J. Macrophages at the fetal-maternal interface express markers of alternative activation and are induced by M-CSF and IL-10. J Immunol (2011) 187(7):3671-82. doi:10.4049/jimmunol

51. Svensson-Arvelund J, Mehta RB, Lindau R, Mirrasekhian E, RodriguezMartinez H, Berg G, et al. The human fetal placenta promotes tolerance against the semiallogeneic fetus by inducing regulatory $\mathrm{T}$ cells and homeostatic M2 macrophages. J Immunol (2015) 194(4):1534-44. doi:10.4049/ jimmunol.1401536 
52. Li M, Piao L, Chen CP, Wu X, Yeh CC, Masch R, et al. Modulation of decidual macrophage polarization by macrophage colony-stimulating factor derived from first-trimester decidual cells: implication in preeclampsia. Am J Pathol (2016) 186(5):1258-66. doi:10.1016/j.ajpath.2015.12.021

53. Huang SJ, Chen CP, Schatz F, Rahman M, Abrahams VM, Lockwood CJ. Preeclampsia is associated with dendritic cell recruitment into the uterine decidua. J Pathol (2008) 214(3):328-36. doi:10.1002/path.2257

54. Huang SJ, Zenclussen AC, Chen CP, Basar M, Yang H, Arcuri F, et al. The implication of aberrant GM-CSF expression in decidual cells in the pathogenesis of preeclampsia. Am J Pathol (2010) 177(5):2472-82. doi:10.2353/ ajpath.2010.091247

55. Wu ZM, Yang H, Li M, Yeh CC, Schatz F, Lockwood CJ, et al. Proinflammatory cytokine-stimulated first trimester decidual cells enhance macrophage-induced apoptosis of extravillous trophoblasts. Placenta (2012) 33(3):188-94. doi:10.1016/j.placenta.2011.12.007

56. Nair RR, Verma P, Singh K. Immune-endocrine crosstalk during pregnancy. Gen Comp Endocrinol (2017) 242:18-23. doi:10.1016/j.ygcen.2016.03.003

57. Robinson DP, Klein SL. Pregnancy and pregnancy-associated hormones alter immune responses and disease pathogenesis. Horm Behav (2012) 62(3):263-71. doi:10.1016/j.yhbeh.2012.02.023

58. Bouman A, Heineman MJ, Faas MM. Sex hormones and the immune response in humans. Hum Reprod Update (2005) 11(4):411-23. doi:10.1093/ humupd/dmi008

59. Soldan SS, Alvarez Retuerto AL, Sicotte NL, Voskuhl RR. Immune modulation in multiple sclerosis patients treated with pregnancy hormone estriol. J Immunol (2003) 171:6267-74. doi:10.4049/jimmuol.171.11.6267

60. Voskuhl R. Sex differences in autoimmune diseases. Biol Sex Differ (2011) 2:1. doi:10.1186/2042-6410-2-1

61. Gellersen B, Brosens JJ. Cyclic decidualization of the human endometrium in reproductive health and failure. Endocr Rev (2014) 35(6):851-905. doi:10.1210/er.2014-1045

62. Arrowsmith S, Kendrick A, Wray S. Drugs acting on the pregnant uterus. Obstet Gynaecol Reprod Med (2010) 20(8):241-7. doi:10.1016/ j.ogrm.2010.05.001

63. Miller L, Alley EW, Murphy WJ, Russell SW, Hunt JS. Progesterone inhibits inducible nitric oxide synthase gene expression and nitric oxide production in murine macrophages. J Leukoc Biol (1996) 59:442-50.

64. Furcron AE, Romero R, Plazyo O, Unkel R, Xu Y, Hassan SS, et al. Vaginal progesterone, but not $17 \alpha$-hydroxyprogesterone caproate, has anti-inflammatory effects at the murine maternal-fetal interface. Am J Obstet Gynecol (2015) 213(6):846.e1-846.e19. doi:10.1016/j.ajog.2015.08.010

65. Unal ER, Cierny JT, Roedner C, Newman R, Goetzl L. Maternal inflammation in spontaneous term labor. Am J Obstet Gynecol (2011) 204(3):223.e221-225. doi:10.1016/j.ajog.2011.01.002

66. Romero R, Dey SK, Fisher SJ. Preterm labor: one syndrome, many causes. Science (2014) 345(6198):760-5. doi:10.1126/science.1251816

67. Kovalevskaya G, Genbacev O, Fisher SJ, Caceres E, O'Connor JF. Trophoblast origin of hCG isoforms: cytotrophoblasts are the primary source of choriocarcinoma-like hCG. Mol Cell Endocrinol (2002) 194(1-2):147-55. doi:10.1016/s0303-7270(02)00135-1

68. Cole LA. Biological functions of hCG and hCG-related molecules. Reprod Biol Endocrinol (2010) 8:102. doi:10.1186/1477-78278-102

69. Lei ZM, Reshef E, Rao V. The expression of human chorionic gonadotropin/ luteinizing hormone receptors in human endometrial and myometrial blood vessels. JClin Endocrinol Metab (1992) 75(2):651-9. doi:10.1210/ jcem.75.2.1379262

70. Licht P, Cao H, Lei ZM, Rao CV, Merz WE. Novel self-regulation of human chorionic gonadotropin biosynthesis in term pregnancy human placenta. Endocrinology (1993) 133(6):3014-25. doi:10.1210/endo.133.6.8243330

71. Zhang YM, Rao Ch V, Lei ZM. Macrophages in human reproductive tissues contain luteinizing hormone/chorionic gonadotropin receptors. Am J Reprod Immunol (2003) 49(2):93-100. doi:10.1034/j.1600-0897.2003. 00013.x

72. Lin J, Lojun S, Lei ZM, Wu WX, Peiner SC, Rao CV. Lymphocytes from pregnant women express human chorionic gonadotropin/luteinizing hormone receptor gene. Mol Cell Endocrinol (1995) 111(1):R13-7. doi:10.1016/ 0303-7207(95)03565-O

73. Herman A, Raziel A, Strassburger D, Soffer Y, Bukovsky I, Ron-El R. The benefits of mid-luteal addition of human chorionic gonadotrophin in in-vitro fertilization using a down-regulation protocol and luteal support with progesterone. Hum Reprod (1996) 11(7):1552-7. doi:10.1093/oxfordjournals. humrep.a019436

74. Zygmunt M, Herr F, Keller-Schoenwetter S, Kunzi-Rapp K, Munstedt $\mathrm{K}$, Rao CV, et al. Characterization of human chorionic gonadotropin as a novel angiogenic factor. J Clin Endocrinol Metab (2002) 87(11):5290-6. doi:10.1210/jc.2002-020642

75. Shi QJ, Lei ZM, Rao CV, Lin J. Novel role of human chorionic gonadotropin in differentiation of human cytotrophoblasts. Endocrinology (1993) 132(3):1387-95. doi:10.1210/endo.132.3.7679981

76. Slattery MM, Brennan C, O'Leary MJ, Morrison JJ. Human chorionic gonadotrophin inhibition of pregnant human myometrial contractility. BJOG (2001) 108(7):704-8. doi:10.1111/j.1471-0528.2001.00178.x

77. Kayisli UA, Selam B, Guzeloglu-Kayisli O, Demir R, Arici A. Human chorionic gonadotropin contributes to maternal immunotolerance and endometrial apoptosis by regulating Fas-Fas ligand system. J Immunol (2003) 171(5):2305-13. doi:10.4049/jimmunol.171.5.2305

78. Sonoda N, Katabuchi H, Tashiro H, Ohba T, Nishimura R, Minegishi T, et al. Expression of variant luteinizing hormone/chorionic gonadotropin receptors and degradation of chorionic gonadotropin in human chorionic villous macrophages. Placenta (2005) 26(4):298-307. doi:10.1016/j.placenta. 2004.07.001

79. Furcron AE, Romero R, Mial TN, Balancio A, Panaitescu B, Hassan SS, et al. Human chorionic gonadotropin has anti-inflammatory effects at the maternal-fetal interface and prevents endotoxin-induced preterm birth, but causes dystocia and fetal compromise in mice. Biol Reprod (2016) 94(6):136. doi:10.1095/biolreprod.116.139345

80. Radtke F, Fasnacht N, Macdonald HR. Notch signaling in the immune system. Immunity (2010) 32(1):14-27. doi:10.1016/j.immuni.2010.01.004

81. Perez-Cabezas B, Naranjo-Gomez M, Bastos-Amador P, Requena-Fernandez G, Pujol-Borrell R, Borras FE. Ligation of Notch receptors in human conventional and plasmacytoid dendritic cells differentially regulates cytokine and chemokine secretion and modulates Th cell polarization. J Immunol (2011) 186(12):7006-15. doi:10.4049/jimmunol.1100203

82. Wang YC, He F, Feng F, Liu XW, Dong GY, Qin HY, et al. Notch signaling determines the M1 versus M2 polarization of macrophages in antitumor immune responses. Cancer Res (2010) 70(12):4840-9. doi:10.1158/00085472.CAN-10-0269

83. Agrawal V, Jaiswal MK, Pamarthy S, Katara GK, Kulshrestha A, GilmanSachs A, et al. Role of Notch signaling during lipopolysaccharide-induced preterm labor. J Leukoc Biol (2015) 100(2):261-74. doi:10.1189/jlb.3HI0515200RR

84. Jaiswal MK, Agrawal V, Pamarthy S, Katara GK, Kulshrestha A, GilmanSachs A, et al. Notch signaling in inflammation-induced preterm labor. Sci Rep (2015) 5:15221. doi:10.1038/srep15221

85. Kong L, Zhang Q, Chao J, Wen H, Zhang Y, Chen H, et al. Polarization of macrophages induced by Toxoplasma gondii and its impact on abnormal pregnancy in rats. Acta Trop (2015) 143:1-7. doi:10.1016/j.actatropica.2014.12.001

86. Xu X, Liu T, Zhang A, Huo X, Luo Q, Chen Z, et al. Reactive oxygen species-triggered trophoblast apoptosis is initiated by endoplasmic reticulum stress via activation of caspase-12, CHOP, and the JNK pathway in Toxoplasma gondii infection in mice. Infect Immun (2012) 80(6):2121-32. doi:10.1128/IAI.06295-11

87. Zhou DH, Yuan ZG, Zhao FR, Li HL, Zhou Y, Lin RQ, et al. Modulation of mouse macrophage proteome induced by Toxoplasma gondii tachyzoites in vivo. Parasitol Res (2011) 109(6):1637-46. doi:10.1007/s00436-0112435-z

88. Jensen KD, Wang Y, Wojno ED, Shastri AJ, Hu K, Cornel L, et al. Toxoplasma polymorphic effectors determine macrophage polarization and intestinal inflammation. Cell Host Microbe (2011) 9(6):472-83. doi:10.1016/ j.chom.2011.04.015

89. Taylor S, Barragan A, Su C, Fux B, Fentress SJ, Tang K, et al. A secreted serine-threonine kinase determines virulence in the eukaryotic pathogen Toxoplasma gondii. Science (2006) 314(5806):1776-80. doi:10.1126/ science. 1133643

90. Rosowski EE, Lu D, Julien L, Rodda L, Gaiser RA, Jensen KD, et al. Strainspecific activation of the NF-kappaB pathway by GRA15, a novel Toxoplasma gondii dense granule protein. J Exp Med (2011) 208(1):195-212. doi:10.1084/ jem. 20100717 
91. Yamamoto M, Standley DM, Takashima S, Saiga H, Okuyama M, Kayama H, et al. A single polymorphic amino acid on Toxoplasma gondii kinase ROP16 determines the direct and strain-specific activation of Stat3. J Exp Med (2009) 206(12):2747-60. doi:10.1084/jem.20091703

92. Murray PJ. Macrophages as a battleground for Toxoplasma pathogenesis. Cell Host Microbe (2011) 9(6):445-7. doi:10.1016/j.chom.2011.05.010

93. Li M, Mo XW, Wang L, Chen H, Luo QL, Wen HQ, et al. Phylogeny and virulence divergency analyses of Toxoplasma gondii isolates from China. Parasit Vectors (2014) 7:133. doi:10.1186/1756335-7-133

94. Hunt JS, Langat DL. HLA-G: a human pregnancy-related immunomodulator. Curr Opin Pharmacol (2009) 9(4):462-9. doi:10.1016/j.coph.2009.05.007

95. Morales PJ, Pace JL, Platt JS, Phillips TA, Morgan K, Fazleabas AT, et al. Placental cell expression of HLA-G2 isoforms is limited to the invasive trophoblast phenotype. J Immunol (2003) 171(11):6215-24. doi:10.4049/ jimmunol.171.11.6215

96. Steinborn A, Varkonyi T, Scharf A, Bahlmann F, Klee A, Sohn C. Early detection of decreased soluble HLA-G levels in the maternal circulation predicts the occurrence of preeclampsia and intrauterine growth retardation during further course of pregnancy. Am J Reprod Immunol (2007) 57(4):277-86. doi:10.1111/j.1600-0897.2007.00475.x

97. Mor G, Abrahams VM. Potential role of macrophages as immunoregulators of pregnancy. Reprod Biol Endocrinol (2003) 1:119. doi:10.1186/1477-7827-1119

98. Li C, Houser BL, Nicotra ML, Strominger JL. HLA-G homodimerinduced cytokine secretion through HLA-G receptors on human decidual macrophages and natural killer cells. Proc Natl Acad Sci U S A (2009) 106(14):5767-72. doi:10.1073/pnas.0901173106

99. Lee CL, Guo Y, So KH, Vijayan M, Guo Y, Wong VH, et al. Soluble human leukocyte antigen G5 polarizes differentiation of macrophages toward a decidual macrophage-like phenotype. Hum Reprod (2015) 30(10):2263-74. doi:10.1093/humrep/dev196

100. Abomaray FM, Al Jumah MA, Kalionis B, AlAskar AS, Al Harthy S, Jawdat D, et al. Human chorionic villous mesenchymal stem cells modify the functions of human dendritic cells, and induce an anti-Inflammatory phenotype in CD1+ dendritic cells. Stem Cell Rev (2015) 11(3):423-41. doi:10.1007/ s12015-0149562-8

101. Insausti CL, Blanquer M, Garcia-Hernandez AM, Castellanos G, Moraleda JM. Amniotic membrane-derived stem cells: immunomodulatory properties and potential clinical application. Stem Cells Cloning (2014) 7:53-63. doi:10.2147/SCCAA.S58696

102. Vacca P, Montaldo E, Vitale C, Croxatto D, Moretta L, Mingari MC. MSC and innate immune cell interactions: a lesson from human decidua. Immunol Lett (2015) 168(2):170-4. doi:10.1016/j.imlet.2015.05.006

103. Parolini O, Alviano F, Bagnara GP, Bilic G, Buhring HJ, Evangelista M, et al. Concise review: isolation and characterization of cells from human term placenta: outcome of the first international workshop on placenta derived stem cells. Stem Cells (2008) 26(2):300-11. doi:10.1634/stemcells.2007-0594

104. Marcus AJ, Woodbury D. Fetal stem cells from extra-embryonic tissues: do not discard. J Cell Mol Med (2008) 12(3):730-42. doi:10.1111/ j.1582-4934.2008.00221.x

105. Pianta S, Bonassi Signoroni P, Muradore I, Rodrigues MF, Rossi D, Silini A, et al. Amniotic membrane mesenchymal cells-derived factors skew $\mathrm{T}$ cell polarization toward Treg and downregulate Th1 and Th17 cells subsets. Stem Cell Rev (2015) 11(3):394-407. doi:10.1007/s12015-014-9558-4

106. Kronsteiner B, Peterbauer-Scherb A, Grillari-Voglauer R, Redl H, Gabriel C, van Griensven $\mathrm{M}$, et al. Human mesenchymal stem cells and renal tubular epithelial cells differentially influence monocyte-derived dendritic cell differentiation and maturation. Cell Immunol (2011) 267(1):30-8. doi:10.1016/ j.cellimm.2010.11.001

107. Magatti M, Caruso M, De Munari S, Vertua E, De D, Manuelpillai U, et al. Human amniotic membrane-derived mesenchymal and epithelial cells exert different effects on monocyte-derived dendritic cell differentiation and function. Cell Transplant (2015) 24(9):1733-52. doi:10.3727/ $096368914 X 684033$

108. Pianta S, Magatti M, Vertua E, Bonassi Signoroni P, Muradore I, Nuzzo AM, et al. Amniotic mesenchymal cells from pre-eclamptic placentae maintain immunomodulatory features as healthy controls. J Cell Mol Med (2016) 20(1):157-69. doi:10.1111/jcmm.12715

109. Monney L, Sabatos CA, Gaglia JL, Ryu A, Waldner H, Chernova T, et al. Th1-specific cell surface protein Tim-3 regulates macrophage activation and severity of an autoimmune disease. Nature (2002) 415(6871):536-41. doi:10.1038/415536a

110. Zhu C, Anderson AC, Schubart A, Xiong H, Imitola J, Khoury SJ, et al. The Tim-3 ligand galectin-9 negatively regulates T helper type 1 immunity. Nat Immunol (2005) 6(12):1245-52. doi:10.1038/ni1271

111. Anderson AC, Anderson DE, Bregoli L, Hastings WD, Kassam N, Lei C, et al. Promotion of tissue inflammation by the immune receptor Tim-3 expressed on innate immune cells. Science (2007) 318(5853):1141-3. doi:10.1126/ science. 1148536

112. Nakayama M, Akiba H, Takeda K, Kojima Y, Hashiguchi M, Azuma M, et al. Tim-3 mediates phagocytosis of apoptotic cells and cross-presentation. Blood (2009) 113(16):3821-30. doi:10.1182/blood-2008-10-185884

113. Meggyes M, Miko E, Polgar B, Bogar B, Farkas B, Illes Z, et al. Peripheral blood TIM-3 positive NK and CD8+ T cells throughout pregnancy: TIM-3/ galectin-9 interaction and its possible role during pregnancy. PLoS One (2014) 9(3):e92371. doi:10.1371/journal.pone.0092371

114. Chabtini L, Mfarrej B, Mounayar M, Zhu B, Batal I, Dakle PJ, et al. TIM-3 regulates innate immune cells to induce fetomaternal tolerance. J Immunol (2013) 190(1):88-96. doi:10.4049/jimmunol.1202176

115. Takahashi K, Naito M, Katabuchi H, Higashi K. Development, differentiation, and maturation of macrophages in the chorionic villi of mouse placenta with special reference to the origin of Hofbauer cells. J Leukoc Biol (1991) 50(1):5768.

116. Karakaya YA, Ozer E. The role of Hofbauer cells on the pathogenesis of early pregnancy loss. Placenta (2013) 34(12):1211-5. doi:10.1016/ j.placenta.2013.10.010

117. Bockle BC, Solder E, Kind S, Romani N, Sepp NT. DC-sign+ CD163+ macrophages expressing hyaluronan receptor LYVE-1 are located within chorion villi of the placenta. Placenta (2008) 29(2):187-92. doi:10.1016/ j.placenta.2007.11.003

118. Joerink M, Rindsjo E, van Riel B, Alm J, Papadogiannakis N. Placental macrophage (Hofbauer cell) polarization is independent of maternal allergen-sensitization and presence of chorioamnionitis. Placenta (2011) 32:380-5. doi:10.1016/j.placenta.2007.11.003

119. Samaniego R, Palacios BS, Domiguez-Soto A, Vidal C, Salas A, Matsuyama $\mathrm{T}$, et al. Macrophage uptake and accumulation of folates are polarizationdependent in vitro and in vivo and are regulated by activin A. J Leukoc Biol (2014) 95(5):797-808. doi:10.1189/jlb.0613345

120. Tang Z, Buhimschi IA, Buhimschi CS, Tadesse S, Norwitz E, Niven-Fairchild $\mathrm{T}$, et al. Decreased levels of folate receptor-beta and reduced numbers of fetal macrophages (Hofbauer cells) in placentas from pregnancies with severe pre-eclampsia. Am J Reprod Immunol (2013) 70(2):104-15. doi:10.1111/ aji. 12112

121. Radaelli T, Varastehpour A, Catalano P, Hauguel-de Mouzon S. Gestational diabetes induces placental genes for chronic stress and inflammatory pathways. Diabetes (2003) 52(2):2951-8. doi:10.2337/diabetes.52.12.2951

122. Sisino G, Bouckenooghe T, Aurientis S, Fontaine P, Storme L, Vambergue A. Diabetes during pregnancy influences Hofbauer cells, a subtype of placental macrophages, to acquire a pro-inflammatory phenotype. Biochim Biophys Acta (2013) 1832(12):1959-68. doi:10.1016/j.bbadis.2013. 07.009

123. Chen W, Wang J, Jia L, Liu J, Tian Y. Attenuation of the programmed cell death-1 pathway increases the M1 polarization of macrophages induced by zymosan. Cell Death Dis (2016) 7(2):e2115. doi:10.1038/cddis. 2016.33

124. Yao A, Liu F, Chen K, Tang L, Liu L, Zhang K, et al. Programmed death 1 deficiency induces the polarization of macrophages/microglia to the M1 phenotype after spinal cord injury in mice. Neurotherapeutics (2014) 11(3):636-50. doi:10.1007/s13311-013-0254-X

125. Fang S, Xu Y, Zhang Y, Tian J, Li J, Li Z, et al. Irgm1 promotes M1 but not M2 macrophage polarization in atherosclerosis pathogenesis and development. Atherosclerosis (2016) 251:282-90. doi:10.1016/j.atherosclerosis. 2016.07.011

126. Wu XQ, Dai Y, Yang Y, Huang C, Meng XM, Wu BM, et al. Emerging role of microRNAs in regulating macrophage activation and polarization in immune response and inflammation. Immunology (2016) 148(3):237-48. doi:10.1111/imm.12608

127. PatelU, Rajasingh S, SamantaS, Cao T, Dawn B, RajasinghJ. Macrophage polarization in response to epigenetic modifiers during infection and inflammation. 
Drug Discov Today (2017) 22(1):186-93. doi:10.1016/j.drudis.2016. 08.006

128. Thangavel J, Samanta S, Rajasingh S, Barani B, Xuan YT, Dawn B, et al. Epigenetic modifiers reduce inflammation and modulate macrophage phenotype during endotoxemia-induced acute lung injury. J Cell Sci (2015) 128(16):3094-105. doi:10.1242/jcs.170258

129. Wang Y, Wang YP, Zheng G, Lee VW, Ouyang L, Chang DH, et al. Ex vivo programmed macrophages ameliorate experimental chronic inflammatory renal disease. Kidney Int (2007) 72(3):290-9. doi:10.1038/ sj.ki.5002275101
Conflict of Interest Statement: The authors declare that the research was conducted in the absence of any commercial or financial relationships that could be construed as a potential conflict of interest.

Copyright $\odot 2017$ Zhang, He, Wang and Liao. This is an open-access article distributed under the terms of the Creative Commons Attribution License (CC BY). The use, distribution or reproduction in other forums is permitted, provided the original author(s) or licensor are credited and that the original publication in this journal is cited, in accordance with accepted academic practice. No use, distribution or reproduction is permitted which does not comply with these terms. 